\title{
Unsteady mixed convection stagnation-point flow over a plate moving along the direction of flow impingement
}

\begin{abstract}
Purpose: The purpose of this paper is to present the results of an analysis performed to study unsteady mixed convection at the stagnation point flow over a plate moving along the direction of flow impingement. The similarity transformations are used to transform the governing nonlinear partial differential equation to a system of an ordinary differential equation.
\end{abstract}

Design/methodology/approach: The transformed equations are then solved numerically by a shooting technique together with bvp4c function.

Findings: The numerical results are compared with the corresponding results from previous researchers. The effects of the unsteadiness Parameter A, Prandtl number Pr, mixed convection parameter $\lambda$ for plane $(\mathrm{m}=0)$ and axisymmetric $(\mathrm{m}=1)$ flow on the shear stress or the skin friction and heat transfer coefficients, as well as the velocity and temperature profiles, are presented and discussed.

Originality/value: Dual solutions for the opposing flow and multiple solutions for the assisting flow are found

Keyword: Numerical solution; Moving plate; Stagnation point flow; Unsteady mixed convection flow 